\title{
Supply Chain Quality Performance Evaluation Model Based on Intuitionistic Fuzzy PROMETHEE
}

\author{
He Dubo ${ }^{1, a}$, Huang Dong ${ }^{2, b}$ \\ ${ }^{1}$ Department of Management Engineering and Equipment Economy, Naval University of Engineering, Wuhan, China \\ ${ }^{2}$ Department of Management Engineering and Equipment Economy, Naval University of Engineering, Wuhan, China
}

\begin{abstract}
Aiming at the ambiguity and uncertainty in subjective group decision-making of supply chain quality performance evaluation, the traditional preference ranking organization methods for enrichment evaluation (PROMETHEE) is extended to a fuzzy environment, and a new intuitive fuzzy PROMETHEE model is proposed. The model uses intuitionistic fuzzy numbers to express the decision-maker's semantic evaluation information, and establishes a trust function based on the evaluation information to determine the decision-maker's weight. Then, use the method of maximizing the net flow to obtain the attribute weight. Finally, gather the evaluation information to obtain the decision group's pros and cons. The validity and feasibility of the model is verified by an example.
\end{abstract}

\section{Introduction}

As the process of economic globalization is accelerating, the professional division of labor is becoming more and more detailed, and the manufacturing of most parts of products is shifting to supporting enterprises. The proportion of outsourced products in the supply chain has increased, supply bodies have become increasingly diversified, and various quality risks implied in the supply chain have been increasing. Establishing a scientific and effective supply chain quality performance evaluation system and method, and systematically evaluating the quality performance of the supply chain, are of great significance for the identification of potential problems in the supply chain, optimization of the structure, improvement of operating efficiency, and realization of quality goals. Quality performance is a qualitative evaluation and quantitative characteristic quantity that an organization uses to represent its products/services, systems, processes, and operations in its quality operations and results [1]. In actual evaluation, in order to accurately describe the uncertainty in the supply chain system, according to actual needs, decision makers often use semantic variables or fuzzy numbers to express their evaluation information. Different evaluation information will be aggregated to obtain credible group decision information. On the one hand, whether the information of different decision makers can be reasonably expressed and aggregated will directly affect the data foundation of the evaluation model implementation; on the other hand, whether the importance of different evaluation indicators can be effectively determined will greatly affect the effectiveness of the evaluation model.

The preference ranking organization methods for enrichment evaluation (PROMETHEE) is a new multiattribute decision-making method which can scientifically and reasonably evaluate the quality performance of the supply chain. reference [2] enhances the PROMETHEE by integrating with a conflict analysis to solve general multiple-criteria decision-making problems with both quantitative and qualitative criteria. Reference [3] develops a novel PROMETHEE using a Pythagorean fuzzy combinative distance-based precedence approach under complex uncertainty based on Pythagorean fuzzy sets. Reference [4] proposes a preference of green suppliers using the PROMETHEE under the usual criterion preference functions. Because intuitionistic fuzzy sets take into account both membership, non-membership and hesitation, its advantage over traditional fuzzy sets is that it can better characterize the uncertainty in decision makers' evaluation. First, this paper introduces the intuitionistic fuzzy set theory into traditional PROMETHEE, and establishes a decision weight model that takes the difference and uncertainty of decision information as the objective function to determine the objective decision weights of different decision makers. The sum of the net flow gap of the decision maker is maximized to solve the attribute weights. Finally, the pros and cons of different evaluation schemes are obtained by aggregating group decision information and attribute weights. 


\section{Intuitionistic Fuzzy PROMETHEE Group Decision Model}

Preference ranking organization methods for enrichment evaluation (PROMETHEE) is a more commonly used multi-attribute decision-making method proposed by Brans. According to the evaluation value, attribute weight and preference function given by the decision maker, this method obtains the inflow, outflow and net flow of each scheme by pairwise comparison of the attribute values under each attribute between two schemes, and the order of pros and cons of the scheme is obtained according to the order of the net traffic. The traditional PROMETHEE method is mainly used for single-person decision-making, and there is no objective calculation method for attribute weights and decisionmaker weights. Therefore, this article will extend the traditional PROMETHEE method.

\subsection{Flow measurement method}

In order to better reflect the hesitation and uncertainty of decision makers in the evaluation, the intuitive fuzzy set theory is introduced in the first place, which is defined as follows:

Definition 1. Let $X$ be a non-empty set, $A=\left\{\left\langle x, t_{A}(x), f_{A}(x)\right\rangle \mid x \in X\right\}$ is called an intuitionistic fuzzy set, $t_{A}(x)$ and $f_{A}(x)$ are the membership and nonmembership degrees of the elements $x$ in $X$, and $0 \leq t_{A}(x) \leq 1,0 \leq f_{A}(x) \leq 1 . \pi_{A}(x)=1-t_{A}(x)-f_{A}(x)$ is defined as the hesitation of elements $x$ in the intuitionistic fuzzy set $X$ belonging to $X$. The entire intuitionistic fuzzy set $X$ on the non-empty set $X$ is denoted as $\Omega$. In particular, $\tilde{\alpha}=\left(t_{\tilde{\alpha}}, f_{\tilde{\alpha}}, \pi_{\tilde{\alpha}}\right)$ is called intuitionistic fuzzy number (IFN).

Definition 2. Let $\tilde{\alpha}=\left(t_{\tilde{\alpha}}, f_{\tilde{\alpha}}, \pi_{\tilde{\alpha}}\right)$ be an IFN on the nonempty set $X$, then its scoring function and exact function are calculated as:

$$
\begin{gathered}
S_{\tilde{\alpha}}=t_{\tilde{\alpha}}-f_{\tilde{\alpha}} \\
H_{\tilde{\alpha}}=t_{\tilde{\alpha}}+f_{\tilde{\alpha}}
\end{gathered}
$$

Definition 3. Let the intuitionistic fuzzy sets $\tilde{\alpha}_{i}=\left(t_{\tilde{\alpha}_{i}}, f_{\tilde{\alpha}_{i}}, \pi_{\tilde{\alpha}_{i}}\right) \quad(i=1,2)$ be two sets of intuitionistic fuzzy numbers, the size relationship and collation are as follows:

1) If $t_{\tilde{\alpha}_{1}} \geq t_{\tilde{\alpha}_{2}}$ and $f_{\tilde{\alpha}_{1}} \leq f_{\tilde{\alpha}_{2}}$, then $\tilde{\alpha}_{1} \geq \tilde{\alpha}_{2}$.

2) If $t_{\tilde{\alpha}_{1}}=t_{\tilde{\alpha}_{2}}$ and $f_{\tilde{\alpha}_{1}}=f_{\tilde{\alpha}_{2}}$, then $\tilde{\alpha}_{1}=\tilde{\alpha}_{2}$.

3) If $S_{\tilde{\alpha}_{1}}>S_{\tilde{\alpha}_{2}}$, then $\tilde{\alpha}_{1}>\tilde{\alpha}_{2}$.

4) If $S_{\tilde{\alpha}_{1}}=S_{\tilde{\alpha}_{2}}$ and $H_{\tilde{\alpha}_{1}}=H_{\tilde{\alpha}_{2}}$, then $\tilde{\alpha}_{1}=\tilde{\alpha}_{2}$.

5) If $S_{\tilde{\alpha}_{1}}=S_{\tilde{\alpha}_{2}}$ and $H_{\tilde{\alpha}_{1}} \leq H_{\tilde{\alpha}_{2}}$, then $\tilde{\alpha}_{1} \leq \tilde{\alpha}_{2}$.

Definition 4. Let $\tilde{\alpha}_{i}=\left(t_{\tilde{\alpha}_{i}}, f_{\tilde{\alpha}_{i}}, \pi_{\tilde{\alpha}_{i}}\right)(i=1,2)$ be two sets of intuitionistic fuzzy numbers, $\lambda>0$, then

1) $\tilde{\alpha}_{1} \oplus \tilde{\alpha}_{2}=\left(t_{\tilde{\alpha}_{1}}+t_{\tilde{\alpha}_{2}}-t_{\tilde{\alpha}_{1}} t_{\tilde{\alpha}_{2}}, f_{\tilde{\alpha}_{1}} f_{\tilde{\alpha}_{2}},\left(1-t_{\tilde{\alpha}_{1}}\right)\left(1-t_{\tilde{\alpha}_{2}}\right)-f_{\tilde{\alpha}_{1}} f_{\tilde{\alpha}_{2}}\right)$

2) $\tilde{\alpha}_{1} \otimes \tilde{\alpha}_{2}=\left(t_{\tilde{\alpha}_{1}} t_{\tilde{\alpha}_{2}}, f_{\tilde{\alpha}_{1}}+f_{\tilde{\alpha}_{2}}-f_{\tilde{\alpha}_{1}} f_{\tilde{\alpha}_{2}},\left(1-f_{\tilde{\alpha}_{1}}\right)\left(1-f_{\tilde{\alpha}_{2}}\right)-t_{\tilde{\alpha}_{1}} f_{\tilde{\alpha}_{2}}\right)$

3) $\lambda \tilde{\alpha}_{1}=\left(1-\left(1-t_{\tilde{\alpha}_{1}}\right)^{\lambda}, f_{\tilde{\alpha}_{1}}^{\lambda},\left(1-t_{\tilde{\alpha}_{1}}\right)^{\lambda}-f_{\tilde{\alpha}_{1}}^{\lambda}\right)$
Definition 5. The Hamming distance between two intuitionistic fuzzy numbers $\tilde{\alpha}_{i}=\left(t_{\tilde{\alpha}_{i}}, f_{\tilde{\alpha}_{i}}, \pi_{\tilde{\alpha}_{i}}\right)(i=1,2)$ is

$$
d\left(\tilde{\alpha}_{1}, \tilde{\alpha}_{2}\right)=\frac{1}{2}\left(\left|t_{\tilde{\alpha}_{1}}-t_{\tilde{\alpha}_{2}}\right|+\left|f_{\tilde{\alpha}_{1}}-f_{\tilde{\alpha}_{2}}\right|+\left|\pi_{\tilde{\alpha}_{1}}-\pi_{\tilde{\alpha}_{2}}\right|\right)
$$

There are $s$ decision makers $D_{h}(h=1,2, \ldots, S)$ to evaluate the solutions in the solution set $X=\left\{x_{1}, x_{2}, \ldots\right.$, $\left.x_{m}\right\}$, and the evaluation attribute set is $F=\left\{f_{1}, f_{2}, \ldots, f_{n}\right\}$. The evaluation matrix expression given by decision maker $D_{h}$ is as follows:

$$
\tilde{A}^{h}=\left[\tilde{\alpha}_{i j}^{h}\right]_{m \times n}
$$

where $\tilde{\alpha}_{i j}^{h}$ represents the evaluation value in the form of an intuitive fuzzy number given by the decision maker $D_{h}$ to the attribute $f_{j}$ of the scheme $x_{i}$.

Given the preference function in PROMETHEE as follows:

$$
\begin{aligned}
P_{j}^{h}\left(\alpha_{i j}^{h}, \alpha_{k j}^{h}\right) & =\left\{\begin{array}{ll}
d\left(\alpha_{i j}^{h}, \alpha_{k j}^{h}\right) & \alpha_{i j}^{h}>\alpha_{k j}^{h} \\
0 & \alpha_{i j}^{h} \leq \alpha_{k j}^{h}
\end{array}\right. \text { (benefit criteria) (4) } \\
P_{j}^{h}\left(\alpha_{i j}^{h}, \alpha_{k j}^{h}\right) & =\left\{\begin{array}{ll}
d\left(\alpha_{i j}^{h}, \alpha_{k j}^{h}\right) & \alpha_{i j}^{h}<\alpha_{k j}^{h} \\
0 & \alpha_{i j}^{h} \geq \alpha_{k j}^{h}
\end{array}\right. \text { (cost criteria) (5) }
\end{aligned}
$$

where $d\left(\alpha_{i j}^{h}, \alpha_{k j}^{h}\right)$ is the Hamming distance between the two attribute values. The preference function indicates the degree to which the decision maker $D_{h}$ believes that the scheme $x_{i}$ is better than $x_{k}$ in the attribute $f_{j}$.

Assuming $w_{j}$ is the weight of the attribute $f_{j}$, the weighted preference value of the decision maker $D_{h}$ for the scheme $x_{i}$ over $x_{k}$ can be obtained as

$$
\pi^{h}\left(x_{i}, x_{k}\right)=\sum_{j=1}^{n} \omega_{j} P_{j}^{h}\left(\alpha_{i j}^{h}, \alpha_{k j}^{h}\right)
$$

Furthermore, comparing all alternatives with $x_{i}$, the inflow $\Phi_{i}^{h+}$ and outflow $\Phi_{i}^{h-}$ of scheme $x_{i}$ are obtained as

$$
\begin{gathered}
\Phi_{i}^{h+}=\sum_{k=1}^{n} \pi^{h}\left(x_{i}, x_{k}\right)=\sum_{k=1}^{n} \sum_{j=1}^{n} \omega_{j} P_{j}^{h}\left(\alpha_{i j}^{h}, \alpha_{k j}^{h}\right) \\
\Phi_{i}^{h-}=\sum_{k=1}^{n} \pi^{h}\left(x_{k}, x_{i}\right)=\sum_{k=1}^{n} \sum_{j=1}^{n} \omega_{j} P_{j}^{h}\left(\alpha_{k j}^{h}, \alpha_{i j}^{h}\right)
\end{gathered}
$$

where $\Phi_{i}^{h+}$ represents the extent of decision maker $D_{h}$ thinks $x_{i}$ better than other alternatives, $\Phi_{i}^{h-}$ indicates that decision maker $D_{h}$ believes $x_{i}$ inferior to other alternatives. Therefore, the larger the inflow and the smaller the outflow, the better the alternative is over the other schemes. Consider both the inflow and outflow, and compare the pros and cons of the all alternatives through the net flow. The net flow calculation formula is as follows:

$$
\Phi_{i}^{h}=\Phi_{i}^{h+}-\Phi_{i}^{h-}
$$

\subsection{Decision weight determination}

How to determine decision weights has always been an important content in group decision-making. Considering that the greater the degree of deviation of expert evaluation information from group decisionmaking information, the greater the individual cognitive bias, the smaller the decision weight. Since the 
evaluation information of experts is in the form of intuitionistic fuzzy numbers, the degree of uncertainty in expert evaluation can be reflected by the hesitation in intuitionistic fuzzy numbers. The Hamming distance function is introduced to represent the cognitive bias of different decision makers by calculating the Hamming distance between different decision matrices. By definition 4, the Hamming distance between the two decision matrices $\tilde{A}^{h}$ and $\tilde{A}^{k}$ is:

$$
d_{k h}=\sum_{i=1}^{m} \sum_{j=1}^{n} d\left(\tilde{\alpha}_{i j}^{h}, \tilde{\alpha}_{i j}^{k}\right)
$$

Calculate the group decision matrix $\tilde{A}^{*}$ by:

$$
\tilde{A}^{*}=\left[\tilde{\alpha}_{i j}^{*}\right]_{m \times n}=\left[\frac{1}{s}\left(\tilde{\alpha}_{i j}^{1}+\tilde{\alpha}_{i j}^{2}+\cdots+\tilde{\alpha}_{i j}^{s}\right)\right]_{m \times n}
$$

Then the deviation distance of the decision matrix $\tilde{A}^{h}$ of the decision maker $D_{h}$ relative to the group decision matrix $\tilde{A}^{*}$ is

$$
\Delta_{h}=\sum_{i=1}^{m} \sum_{j=1}^{n} d\left(\tilde{\alpha}_{i j}^{h}, \tilde{\alpha}_{i j}^{*}\right)
$$

Considering the consistency requirements, the smaller the deviation distance is, the more reliable its decision information is, and its decision weight should be greater. In order to calculate the expert weights, the normal distribution function is constructed using the deviation and uncertainty of the expert decision matrix, and the variance is:

$$
\sigma^{2}=\frac{1}{s} \sum_{h=1}^{s} \Delta_{h}
$$

the decision maker $D_{h}$ weight probability distribution function is

$$
\rho_{h}=\frac{1}{\sqrt{2 \pi} \sigma} \exp \left(-\frac{d_{h}}{\sigma^{2}}\right)
$$

after normalization, the expert weight is

$$
\tau_{h}=\frac{\rho_{h}}{\sum_{k=1}^{s} \rho_{k}}
$$

the objective expert weight vector is $\tau=\left(\tau_{1}, \tau_{2}, \ldots, \tau_{s}\right)$.

\subsection{Attribute weight determination model}

Because the attribute weights in the evaluation are completely unknown, in order to get the net flow of the solution, the attribute weights must be solved. For the decision maker $D_{h}$, if the difference in net flow between different alternatives are larger, the advantages and disadvantages between the schemes are more obvious. Therefore, the calculation formula of the total net flow gap $\Phi_{h}$ given by $D_{h}$ is as follows:

$$
\Phi_{h}=\sum_{i=1}^{n} \sum_{j>i}^{n}\left|\Phi_{j}^{h}-\Phi_{i}^{h}\right|
$$

Consider the maximum sum of the net flow gap given by each expert in group decision-making to solve the attribute weight value. We can build a multiobjective programming model as follows: $\max \Phi_{1}$

$\max \Phi_{s}$

$$
\text { s.t. }\left\{\begin{array}{l}
\sum_{j=1}^{n} \omega_{j}=1 \\
\omega_{j} \geq 0, j=1,2, \cdots, n .
\end{array}\right.
$$

Based on the decision weight vector $\tau=\left(\tau_{1}, \tau_{2}, \ldots, \tau_{s}\right)$ being known, the expert weight vector can be used to transform the multi-objective programming model into a single-objective programming model as follows:

$$
\begin{aligned}
\max z & =\tau_{1} \Phi_{1}+\tau_{2} \Phi_{2}+\cdots+\tau s \Phi s \\
\text { s.t. } & \left\{\begin{array}{l}
\sum_{j=1}^{n} \omega_{j}=1 \\
\omega_{j} \geq 0, j=1,2, \cdots, n .
\end{array}\right.
\end{aligned}
$$

By solving the single-objective programming model, the weight vector of the evaluation attribute set $\omega=\left(\omega_{1}, \omega_{2}, \cdots, \omega_{n}\right)$ can be obtained. The final net flow value of each scheme to be evaluated

$$
\Phi_{i}=\tau_{1} \Phi_{i}^{1}+\tau_{2} \Phi_{i}^{2}+\cdots+\tau_{s} \Phi_{i}^{s}, i=1,2, \cdots, m .
$$

\section{Case Anaylsis}

Taking the evaluation of the supply chain quality performance of an electronic product as an example, in order to select a suitable supply chain partner, it is necessary to select a supplier with a good supply chain quality performance and a sustainable development strategy. There are 3 suppliers to choose from, denoted as $X=\left\{x_{1}, x_{2}, x_{3}\right\}$, and evaluated by 4 evaluation indicators $F=\left\{f_{1}, f_{2}, f_{3}, f_{4}\right\}$, which are supply chain competitiveness $\left(f_{1}\right)$, supply chain Service quality $\left(f_{2}\right)$, supply chain quality control capability $\left(f_{3}\right)$, supply chain response capability $\left(f_{4}\right)$. These indicators are all benefitoriented. Use the intuitionistic fuzzy PROMETHEE model to evaluate the supply chain quality performance. First, three decision-making experts $\left\{D_{1}, D_{2}, D_{3}\right\}$ are invited to judge the three suppliers based on their own knowledge and experience, as shown in Tables I-III.

TABLE I. EVALUATION MATRIX GIVEN BY EXPERT $D_{1}$

\begin{tabular}{|l|l|l|l|}
\hline \multirow{2}{*}{$\begin{array}{l}\text { Attrib } \\
\text {-utes }\end{array}$} & \multicolumn{4}{|l|}{ Evaluation value of suppliers } \\
\cline { 2 - 4 } & $x 1$ & $x 2$ & $x 3$ \\
\hline$f 1$ & $(0.5,0.3,0.2)$ & $(0.5,0.4,0.1)$ & $(0.7,0.3,0.0)$ \\
\hline$f 2$ & $(0.4,0.3,0.3)$ & $(0.6,0.4,0.0)$ & $(0.2,0.5,0.3)$ \\
\hline$f 3$ & $(0.8,0.2,0.0)$ & $(0.7,0.3,0.0)$ & $(0.4,0.6,0.0)$ \\
\hline$f 4$ & $(0.5,0.4,0.1)$ & $(0.6,0.2,0.2)$ & $(0.5,0.2,0.3)$ \\
\hline
\end{tabular}

TABLE II. EVALUATION MATRIX GIVEN BY EXPERT $D_{2}$

\begin{tabular}{|l|l|l|l|}
\hline \multirow{2}{*}{$\begin{array}{l}\text { Attrib } \\
\text {-utes }\end{array}$} & \multicolumn{3}{|l|}{ Evaluation value of suppliers } \\
\cline { 2 - 4 }$f 1$ & $(0.4,0.3,0.3)$ & $(0.5,0.3,0.2)$ & $(0.6,0.3,0.1)$ \\
\hline$f 1$ & $(0.4,0.6,0.0)$ & $(0.7,0.3,0.0)$ & $(0.3,0.4,0.3)$ \\
\hline$f 3$ & $(0.7,0.3,0.0)$ & $(0.6,0.3,0.1)$ & $(0.4,0.6,0.0)$ \\
\hline$f 4$ & $(0.7,0.2,0.1)$ & $(0.5,0.4,0.1)$ & $(0.9,0.1,0.0)$ \\
\hline
\end{tabular}


TABLE III. EVALUATION MATRIX GIVEN BY EXPERT $D_{3}$

\begin{tabular}{|l|l|l|l|}
\hline \multirow{2}{*}{$\begin{array}{l}\text { Attrib } \\
\text {-utes }\end{array}$} & \multicolumn{3}{|l|}{ Evaluation value of suppliers } \\
\cline { 2 - 4 } & $x 1$ & $x 2$ & $x 3$ \\
\hline$f 1$ & $(0.6,0.3,0.2)$ & $(0.5,0.4,0.1)$ & $(0.5,0.2,0.3)$ \\
\hline$f 2$ & $(0.3,0.3,0.4)$ & $(0.6,0.4,0.0)$ & $(0.2,0.7,0.1)$ \\
\hline$f 3$ & $(0.9,0.1,0.0)$ & $(0.7,0.3,0.0)$ & $(0.4,0.4,0.2)$ \\
\hline$f 4$ & $(0.6,0.2,0.2)$ & $(0.7,0.3,0.0)$ & $(0.4,0.3,0.3)$ \\
\hline
\end{tabular}

According to the formula (11) the expert group decision matrix $\tilde{A}^{*}$ is

$$
\tilde{A}^{*}=\left[\begin{array}{ccc}
(0.523,0.267,0.210) & (0.500,0.367,0.133) & (0.622,0.246,0.132) \\
(0.347,0.420,0.233) & (0.533,0.367,0.100) & (0.256,0.533,0.211) \\
(0.780,0.220,0.000) & (0.637,0.273,0.090) & (0.432,0.522,0.046) \\
(0.644,0.253,0.100) & (0.563,0.356,0.810) & (0.652,0.265,0.083)
\end{array}\right]
$$

According to formula (12), the deviation distances of the decision matrices of different experts and expert groups can be calculated as

$$
\Delta_{1}=1.6333 ; \Delta_{2}=2.7000 ; \Delta_{3}=2.5333
$$

Afterwards, according to the formula (13) (15), the final expert weight vector can be obtained as $\tau=(0.4343,0.2725,0.2931)$. Substituting the expert weight vector into the formula (18) can get the attribute weight vector as $\omega=(0.1375,0.3431,0.2564,0.263)$. Combining the expert weight vector and the attribute weight vector to obtain the net flows of the three evaluation objects are

$$
\Phi_{1}=0.573 ; \Phi_{2}=0.653 ; \Phi_{3}=0.396
$$

Obviously $\Phi_{2}>\Phi_{1}>\Phi_{3}$, so the final result is that supplier $x_{2}$ has the best quality performance in the supply chain, $x_{2}$ is the second, and $x_{3}$ has the worst performance. Both the attribute weight and the expert weight are solved by objective methods, making the final evaluation result more objective and reliable.

\section{Conclusion}

There are many uncertain and fuzzy indicators in supply chain quality performance evaluation that can only be evaluated by subjective group decision making. This paper proposes a multi-attribute group decision model based on intuitionistic fuzzy PERMETHEE by improving traditional PERMETHEE. The use of intuitionistic fuzzy sets to process the expert's semantic evaluation information makes the subjective evaluation information more scientific and reasonable. The objective method of solving expert weights and evaluation attribute weights reduces the subjectivity of the evaluation process. It provides a scientific and effective method for the supply chain quality performance evaluation.

\section{Acknowledgment}

This work is supported by National Social Science Foundation under Grant 19BGL291.

\section{References}

1. W Bangjun, and J Jiadong. (2008) Application of Quality Performance Evaluation Technology in Aviation Industry Enterprises. Aeronautical Standardization and Quality, 1 (05):29-34.

2. Wu, Xingli, et al. (2020) An Integrated Method with PROMETHEE and Conflict Analysis for Qualitative and Quantitative Decision-Making: Case Study of Site Selection for Wind Power Plants. Cognitive Computation,1 (1):1-15

3. Chen, Ting-Yu. (2019) A novel PROMETHEEbased method using a Pythagorean fuzzy combinative distance-based precedence approach to multiple criteria decision making. Applied Soft Computing,82(82).

4. Abdullah, Lazim, W. Chan, and A. Afshari. (2019) Application of PROMETHEE method for green supplier selection: a comparative result based on preference functions. Journal of Industrial Engineering International ,15(2). 